\title{
Adaptive Subpixel Estimation of Land Cover in a Remotely Sensed Multispectral Image
}

\author{
Senya Kiyasu, Kazunori Terashima, Seiji Hotta and Sueharu Miyahara \\ Department of Computer and Information Sciences, Nagasaki University, Nagasaki, Japan \\ (Tel : +81-95-819-2582; E-mail: kiyasu@ cis.nagasaki-u.ac.jp)
}

\begin{abstract}
Land surface corresponding to a pixel of remotely sensed image does not necessarily consist of only one category of objects. Several techniques of subpixel analysis have been developed which estimate the proportion of components of land cover in a pixel. However, when the available training data do not correctly represent the spectral characteristics of the categories in the pixel, large errors may appear in the results of estimation. The method of unsupervised estimation of component spectra has been presented to solve this problem. In this paper we present a method which apply the unsupervised analysis technique to subpixel estimation of land cover in an image in which spectral characteristics change with the location of the objective area. After partitioning the image into blocks, the number of categories and their component spectra are estimated in each block. Then the proportion of category are estimated for each pixel using the component spectra derived in the block. We confirmed the validity of this method by numerical simulation.
\end{abstract}

Keywords: remote sensing, subpixel analysis, component proportion, spectral unmixing, adaptive estimation

\section{INTRODUCTION}

In conventional analysis of remotely sensed images, each pixel is classified into a single object category. However, the actual land surface corresponding to a pixel does not necessarily consist of only one category of objects. Therefore, estimating the proportion of components that exist in a pixel is often useful.

Spectral unmixing is a method by which to estimate the proportion of each component in a pixel using multispectral data. The most commonly used method of spectral unmixing assumes that the component spectra are determined from training data. However, available training data do not always correctly represent the spectral characteristics of the categories within the objective area. In such cases, large errors may appear in the results of unmixing. Some methods of unsupervised estimation of component spectra have already been developed [1-3], and they are promising to be applied to solve this problem.

In this paper we present a method which apply the unsupervised analysis technique to the subpixel estimation of land cover in an image in which spectral characteristics change with the location of the objective area. We estimated the degree of errors by numerical simulation and compared the performance of the proposed method and that of the conventional method.

\section{SUBPIXEL ESTIMATION OF LAND COVER IN A MULTISPECTRAL IMAGE}

Usual method of subpixel estimation is based on the linear mixing model that the observed spectrum is a combination of component spectra weighted by the proportion of components in a pixel $[4,5]$. When a multispectral image is observed at the wavelength $\lambda_{1}, \cdots, \lambda_{L}$, the observed spectrum of $i$-th pixel is expressed as $\boldsymbol{x}_{i}=$ $\left(x_{i 1}, \cdots, x_{i L}\right)^{t}$, where $t$ denotes the transpose of a ma- trix. We assume that $N$ categories of objects exist in the image. Then, the observed vector $\boldsymbol{x}_{i}$ can be written as the linear combinations of $\boldsymbol{s}_{j}$ :

$$
\boldsymbol{x}_{i}=c_{i 1} \boldsymbol{s}_{1}+c_{i 2} \boldsymbol{s}_{2}+\cdots+c_{i N} \boldsymbol{s}_{N}
$$

where $\boldsymbol{s}_{j}=\left(s_{j 1}, \cdots, s_{j L}\right)^{t}$ for $j=1, \cdots, N$ are the component spectra and $c_{i j}$ are the proportions of component $j$ in a pixel $i$. We can write this using matrix as

$$
\boldsymbol{x}_{i}=\boldsymbol{S} \boldsymbol{c}_{i},
$$

where $\boldsymbol{S}$ is a matrix whose columns are component spectra $\boldsymbol{s}_{j}$, and $\boldsymbol{c}_{i}=\left(c_{i 1}, \cdots, c_{i N}\right)^{t}$ is a proportion vector. The proportion of each component is corresponding to the amount of area which the category of object occupies in a pixel. Therefore the proportion vectors $c_{i}$ are limited by the following relations :

$$
\begin{aligned}
& \sum_{j=1}^{N} c_{i j}=1, \\
& c_{i j} \geq 0 .
\end{aligned}
$$

Subpixel analysis is a process of determining $c_{i}$ for given $\boldsymbol{x}_{i}$ by using the component spectra $\boldsymbol{S}$. If the vectors $\boldsymbol{s}_{j}$ are linearly independent, $\boldsymbol{c}_{i}$ can be determined using the component spectra $\boldsymbol{S}$. Conventional method assumes that the component spectra $S$ are known. The component vectors $s_{j}$ are usually derived from a set of training samples. However, they are not always correctly representing the spectral characteristics of components within the object area. If the assumed component spectra $s_{j}$ are not correct, they may cause large errors [6].

\section{PRINCIPLE OF UNSUPERVISED ESTIMATION OF COMPONENT SPECTRA}

In the unsupervised estimation of component spectra[2], the spectral reflectance of pixels is expressed by vectors in multi-dimensional space, which can be written 
as linear combinations of component spectra weighted according to component proportion. We determine the component spectra by finding the minimum volume of simplex containing all of the reflectance vectors, where the vertexes of the simplex correspond to the component spectra (Fig.1).
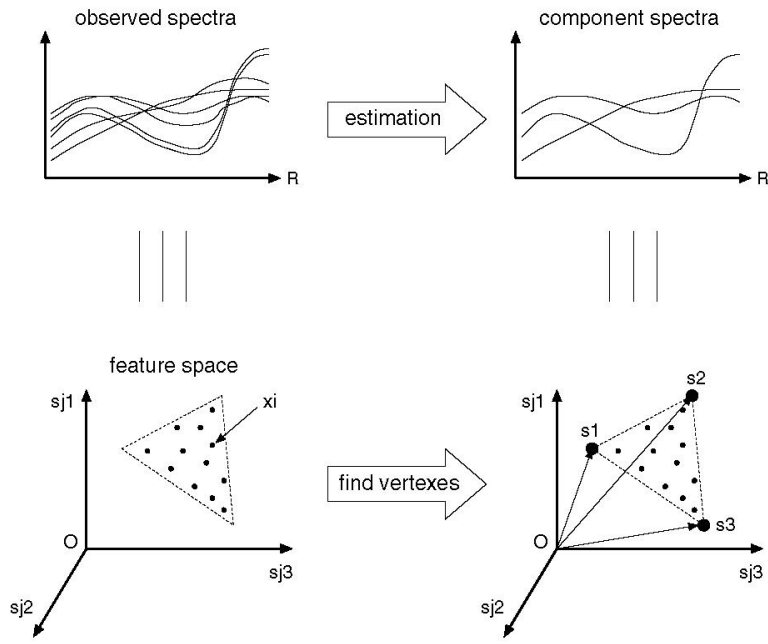

Fig. 1 Estimation of component spectra in the feature space

First we express the component spectra $\boldsymbol{s}_{j}$ by linear combination of eigenvectors $\boldsymbol{v}_{1}, \cdots, \boldsymbol{v}_{N}$, as follows :

$$
\boldsymbol{s}_{j}=t_{j 1} \boldsymbol{v}_{1}+t_{j 2} \boldsymbol{v}_{2}+\cdots+t_{j N} \boldsymbol{v}_{N}
$$

where $t_{j k}$ are coefficients of eigenvectors. The eigenvectors are calculated using the second moment matrix:

$$
\boldsymbol{R}=\sum_{i=1}^{M} \boldsymbol{x}_{i} \boldsymbol{x}_{i}^{t} / M
$$

where $M$ is the number of observed vectors. The eigenvectors span a feature space in which the component spectra $\boldsymbol{s}_{j}$ and the observed vector $\boldsymbol{x}_{i}$ are represented by the points $\boldsymbol{t}_{j}=\left(t_{j 1}, \cdots, t_{j N}\right)^{t}$ and $\boldsymbol{r}_{i}=\left(r_{i 1}, \cdots, r_{i N}\right)^{t}$ respectively, where

$$
\begin{aligned}
& \boldsymbol{t}_{j}=\boldsymbol{V}^{t} \boldsymbol{s}_{j}, \\
& \boldsymbol{r}_{i}=\boldsymbol{V}^{t} \boldsymbol{x}_{i},
\end{aligned}
$$

and $\boldsymbol{V}=\left(\boldsymbol{v}_{1}, \cdots, \boldsymbol{v}_{N}\right)^{t}$. Therefore, vector $\boldsymbol{r}_{i}$ is expressed as

$$
\boldsymbol{r}_{i}=c_{i 1} \boldsymbol{t}_{1}+c_{i 2} \boldsymbol{t}_{2}+\cdots+c_{i N} \boldsymbol{t}_{N},
$$

which means that $\boldsymbol{r}_{i}$ exists inside of the $N-1$ dimensional simplex defined by $\boldsymbol{t}_{1}, \cdots, \boldsymbol{t}_{N}$.

Next we find the minimum volume of simplex which include all the points $r_{i}$ inside of it. We define a object function $U$ and find the vectors $t_{1}, \cdots, \boldsymbol{t}_{N}$ which minimize the function $U$. The object function is defined as

$$
U=S+P \longrightarrow \min ,
$$

were $S$ is the volume of the $N-1$ dimensional simplex and $P$ is the penalty function which expresses the nonnegative constraints of component spectrum and proportion. We used the penalty function $P$ in the same way as Ref.[7] :

$$
P=\gamma\left\{\sum_{j=1}^{N} \sum_{l=1}^{L} F\left(s_{j l}\right)+\sum_{i=1}^{M} \sum_{j=1}^{N} F\left(c_{i j}\right)\right\}
$$

where $\gamma$ is a scaling factor and $F(\cdot)$ is the function

$$
F(x)= \begin{cases}0 & \text { if } x \geq 0 \\ x^{2} & \text { if } x<0\end{cases}
$$

We used the "simplex method" for numerical processing of nonlinear optimization.

After the vectors $\boldsymbol{t}_{j}$ are determined, component spectra $s_{j}$ can be calculated by Eq.(5), and proportion of each component $c_{i}$ can be determined using the generalized inverse matrix as

$$
c_{i}=\left(\boldsymbol{S}^{t} \boldsymbol{S}\right)^{-1} \boldsymbol{S}^{t} \boldsymbol{x}_{i}
$$

\section{ALGORITHM OF ADAPTIVE SUBPIXEL ESTIMATION FOR MULTISPECTRAL IMAGE}

We present here an algorithm of adaptive land cover estimation applying the unsupervised analysis of component spectra.

In remotely sensed images, spectral characteristics of objects sometimes change with the location of the objective area. In such cases, component spectra which derived from specific area not always appropriate for other areas. Therefore, we divided the object image into suitable blocks and repeated the estimation process of component spectra in each block.

\subsection{Partitioning an image}

The first step of the method is to partitioning the multispectral image into blocks as shown in Fig.2. We tried to reduce the influence of the spectral variation with the location by partitioning the image into blocks and estimating the component spectra in each block.

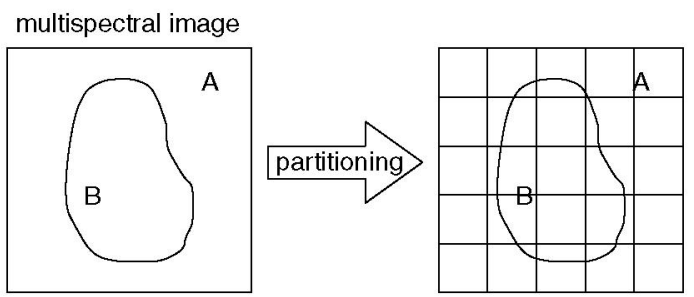

Fig. 2 Partitioning an image into blocks

\subsection{Estimation of components in each block}

The next step is to determine the number of categories and their spectra in the blocks. We assume here that the number of categories exist in the whole image is known and their preliminary spectra can be derived from the image. The method of unsupervised estimation of component spectra can be applied to determine the preliminary spectra of categories in the whole image.

We evaluate the similarity between the preliminary spectra and the observed spectra. We can use the Euclidean distance in a feature space, for example, for a 
measure of similarity. If some of the pixels in the block show similar characteristics with one of the preliminary spectrum, we consider that the category is exist in the objective area. After the existing categories are determined, their accurate spectra are adaptively estimated according to the unsupervised method in each of the blocks.

\subsection{Estimation of land cover in a pixel}

Last step is to calculate the proportion of categories in the pixels and estimate the land cover with subpixel accuracy. The spectra estimated in the former step are used for calculation in each block.

\section{EXPERIMENTS AND RESULTS}

We confirmed the validity of the algorithm by numerical simulation. We used a simulated image of 225 by 225 pixels as shown in Fig. 3 which has three regions of different category. The contiguous categories were assumed to be mixed in the boundary pixels, where the mixture ratio was set to be random for each pixel. The reflectance was set to be increased linearly by $5 \%$ from left to right.

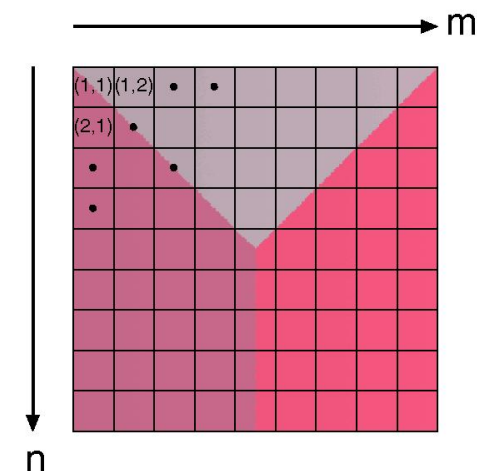

Fig. 3 Simulated image and partitioning format

We used three different categories of hyperspectral data for each pure component. The number of spectral dimension is 220 . The spectral data were derived from a hyperspectral image taken by the AVIRIS (Airborne Visible Infrared Imaging Spectrometer). The three spectra shown in Fig.4 are corresponding to the mean spectra of "grass", "soybeans" and "woods".

The image was partitioned into 9 by 9 blocks as shown in Fig. 3 and processed according to the proposed procedure. Figure 5 shows the feature space in which all pixels within one of the blocks are expressed. Since the number of components is three, the three determined component spectra are indicated by a triangle. It is shown that the component spectra ware accurately estimated. The estimated spectra are also expressed on Fig.4 as plotted points. We can see that they are fit well with the true spectra.

Figure 6 and 7 show the relationship between true and estimated proportion in each of the pixels. Figure 6 shows the result of conventional method and Fig. 7 shows the result of proposed method respectively. We can see that the estimation error was significantly reduced by the proposed method.



Fig. 4 Spectral data for simulation (true and estimated spectra)

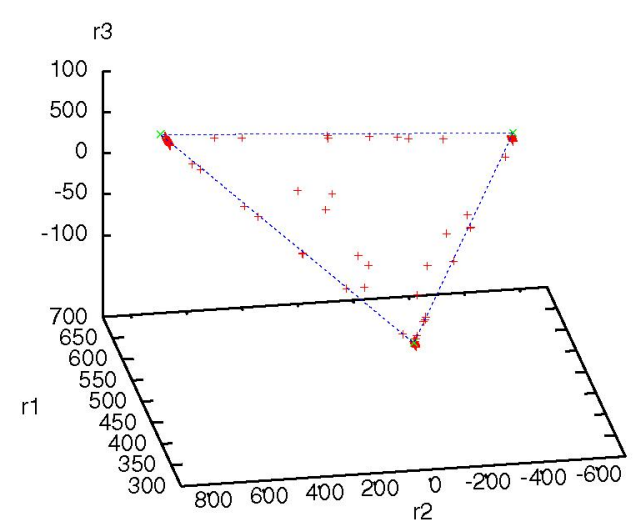

Fig. 5 Feature space for component spectra estimation

\section{CONCLUSION}

We have proposed a method which adaptively estimate the land cover proportion in a pixel of remotely sensed multispectral image. By this method we can determine the spectra of component without training data and estimate the proportion of components adaptively where the spectral characteristics change with the location of the objective area.

Application of this method to remotely sensed real image is the step of the next study.

\section{REFERENCES}

[1] S. Kiyasu, S. Araki, H. Takeuchi and S. Fujimura, "Adaptive Spectral Unmixing for Estimation of Component Proportion," Proc. of ISNIC'98, pp. 239-244, 1998.

[2] S. Kiyasu and S. Fujimura, "Adaptive Estimation of Component Proportion in a Pixel of a Multispectral Image," Trans. of SICE, Vol. 39, No. 2, pp. 97-103, 2003 (in Japanese).

[3] M. E. Winter, "N-FINDR: an algorithm for fast autonomous spectral end-member determination in 


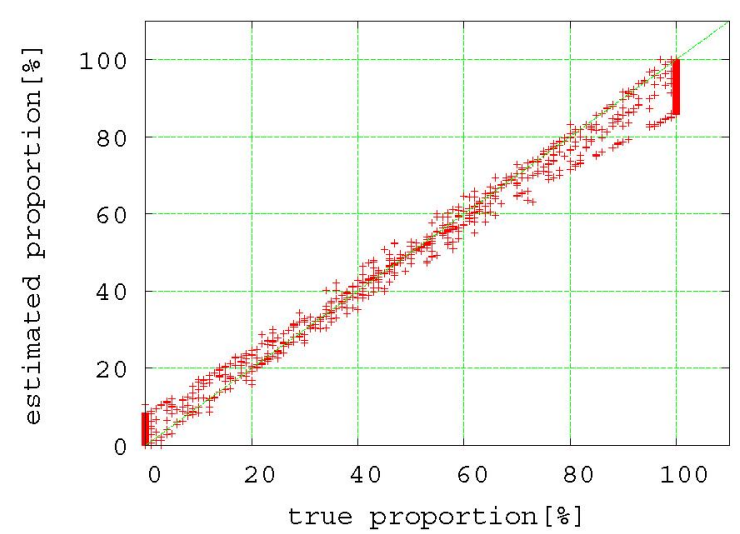

Fig. 6 Error of estimation by conventional method

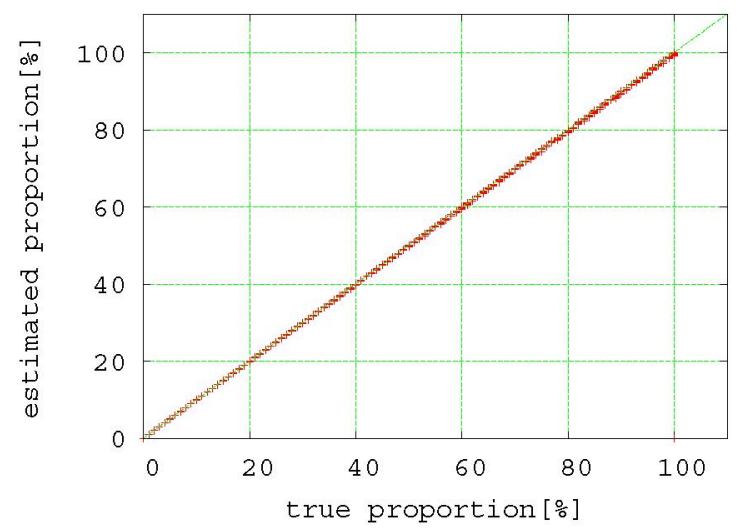

Fig. 7 Error of estimation by proposed method

hyperspectral data," Proc. of SPIE, Vol. 3753, pp. 266-277, 1999.

[4] Minoru Inamura, "Analysis of Remotely Sensed Image Data by means of Category Decomposition," Trans. IEICE, Vol. J-70-C, No2, pp.241-250,1987 (in Japanese).

[5] J.J.Settle and N.A.Drake "Linear mixing and the estimation of ground cover proportions," Int. J. Remote Sensing, Vol.14, No.6, pp.1159-1177, 1993.

[6] Tadashi Ito and Sadao Fujimura, "Estimation of Cover Area of Each Category in a Pixel by Pixel Decomposition into Categories," Trans. SICE, Vol.23, No.8, pp.800-805, 1987 (in Japanese).

[7] K.Sasaki, S.Kawata and S.Minami, "Estimation of Component Spectral Curves from Unknown Mixture Spectra," Appl.Opt., Vol. 23, pp.1955-1959, 1984. 